\title{
Cancer immunotherapy: harnessing the immune system to battle cancer
}

\author{
Yiping Yang \\ Division of Hematologic Malignancies and Cellular Therapy, Department of Medicine, and Department of Immunology, Duke University, Durham, North Carolina, USA
}

\begin{abstract}
The recent clinical successes of immune checkpoint blockade and chimeric antigen receptor $\mathrm{T}$ cell therapies represent a turning point in cancer immunotherapy. These successes also underscore the importance of understanding basic tumor immunology for successful clinical translation in treating patients with cancer. The Reviews in this Review Series focus on current developments in cancer immunotherapy, highlight recent advances in our understanding of basic aspects of tumor immunology, and suggest how these insights can lead to the development of new immunotherapeutic strategies.
\end{abstract}

\section{Introduction}

The concept that the immune system can recognize and control tumor growth can be traced back to 1893 when William Coley used live bacteria as an immune stimulant to treat cancer, but the enthusiasm for cancer immunotherapy has been moderate due to limited clinical efficacy. This limited efficacy is due to the ability of tumor cells to avoid recognition and elimination by the immune system, allowing them to become established in the host (1). Over the past few decades, tremendous progress has been made in the understanding of how cancer evades the immune system, which in turn offers new ways to stop cancer immune evasion in favor of eliminating cancer cells.

The recent clinical success by immune checkpoint therapy, using blocking antibodies to cytotoxic $\mathrm{T}$ lymphocyte antigen-4 (CTLA-4) and programmed death-1 (PD-1), and by chimeric antigen receptor (CAR) $\mathrm{T}$ cells represents the result of efforts to tip the balance in favor of the immune system in the elimination of cancer cells. Clinical trials have shown their potential in lifesaving; as such, "cancer immunotherapy" was named as 2013's Breakthrough of the Year by Science (2). In addition, the success of these therapies illustrates the importance of careful decoding of basic immunology for successful clinical translation in treating cancer. The goal of this Review Series is to concisely review some of the recent developments in cancer immunology and immunotherapy and to detail how new insights into the mechanisms that underlie cancer immune evasion might lead to pathways for identifying novel and efficacious treatments. By framing basic mechanistic studies in a clinical context, we hope that these reviews will be of interest to both practicing oncologists and cancer immunologists.

\section{Immunity, inflammation, and cancer}

In order to delineate the underlying basis for cancer immune evasion and to design effective immunotherapies, it is essential to understand how cancer interacts with the immune system. Accumulating evidence over the last decade from mouse models and

Conflict of interest: The author has declared that no conflict of interest exists. Reference information: / Clin Invest. 2015;125(9):3335-3337. doi:10.1172/JCI83871. human patients with cancer has demonstrated the importance of the immune system in recognizing and eliminating transformed malignant cells. The immune system also plays a critical role in promoting tumor progression. This dual role by which the immune system can suppress and/or promote cancer growth is termed cancer immunoediting and consists of three phases: elimination, equilibrium, and escape (3). In this Review Series, Teng and colleagues review recent developments in cancer immunoediting, particularly the importance of $\mathrm{CD}^{+} \mathrm{T}$ cells in cancer immunoediting and more broadly in tumors with an adaptive immune resistance phenotype (4). They further describe the characteristics of an adaptive immune resistance tumor microenvironment that affect survival outcome, including immune contexture, immunoscore, and the presence of tertiary lymphoid structures. They also discuss the temporal occurrence of cancer immunoediting in metastases. By understanding immunoediting in the tumor microenvironment, key pathways that suppress endogenous antitumor responses can be targeted, leading to potential novel therapies for patients with cancer.

Similar to the dual roles of $\mathrm{T}$ cells in cancer immunoediting, inflammation also has two roles in shaping cancer development. The Review by Shalapour and Karin discusses how tumorrelated chronic inflammation shapes local and systemic immunity to promote an immunosuppressive tumor microenvironment and tumor development, whereas acute inflammation can enhance antitumor immunity by promoting DC maturation and function as well as effector T cell priming (5). Further understanding of how inflammation influences cancer development and progression will lead to novel strategies that enhance antitumor immunity by targeting immunosuppressive chronic inflammation.

\section{Immunosuppressive myeloid cells in tumor microenvironment}

It has been well recognized that growing cancers contain tumor-infiltrating lymphocytes (TILs), which are ineffective at tumor elimination in vivo but can exert proliferation and effector function when removed from the immunosuppressive tumor microenvironment. This is because cancer cells have developed mechanisms to avoid recognition and elimination by the immune system 
(1). The major mechanisms by which tumors evade destruction by the immune system include downmodulation of components of antigen processing and presentation machinery; recruitment of suppressor immune cells, such as regulatory $\mathrm{T}$ cells, myeloidderived suppressor cells (MDSCs), and tumor-associated macrophages; production of soluble factors associated with immunosuppression, such as TGF- $\beta$ and IL-10; and upregulation of ligands for coinhibitory receptors that downmodulate TIL activity, such as programmed death ligand-1 (PD-L1).

Marvel and Gabrilovich review recent developments in the role of MDSCs in cancer (6). They explain that, in addition to immunosuppression, MDSCs can directly support tumor growth and metastasis. They also discuss the most pertinent issues of MDSC biology and highlight how these cells may be used both as prognostic factors and as therapeutic targets clinically. The Review by Ugel and colleagues covers tumor-associate myeloid cells, including MDSCs and tumor-associated macrophages (7). They explain how tumor cells reprogram myeloid cells to create an immunosuppressive environment as well as to drive tumor progression directly by promoting cancer stemness, angiogenesis, epithelial-to-mesenchymal transition, and metastasis. They also discuss the molecular pathways leading to the differentiation of tumor-programmed myeloid cells and their potential roles as prognostic/diagnostic biomarkers and therapeutic targets in the clinic.

\section{Immune checkpoint blockade therapy}

The elucidation of mechanisms underlying cancer immune regulation has been instrumental in the recent success of immune checkpoint therapy using antibodies that block CTLA-4 and PD-1 pathways to treat patients with cancer. CTLA- 4 was discovered by Pierre Goldstein in 1987 (8). Later, several groups independently proved that CTLA-4 functions as an inhibitory receptor both in vitro and in knockout mice (9-12). These discoveries led to James Allison's seminal work in 1996, demonstrating that CTLA-4 blockade erased tumors in mice (13), which provided a rationale for the subsequent clinical development of CTLA-4-targeting antibodies. In 2011, the US Food and Drug Administration approved antiCTLA-4 antibodies (ipilimumab) for use in treating melanoma, which marked the beginning of a new era for cancer immunotherapy. In this Review Series, Buchbinder and Hodi review the current state of anti-CTLA-4 therapy, including clinical efficacy, associated toxicities, and combinatorial strategies with radiation therapy, chemotherapy, and PD-1 blockade to increase the efficacy of anti-CTLA-4 therapy (14).

The clinical development of PD-1 blockade was dependent on a sequence of basic science discoveries. PD-1 was originally cloned by Tasuku Honjo in 1992 (15). Almost 10 years later, the ligand for PD-1 (PD-L1) was found independently by two research groups led by Lieping Chen and Gordon Freeman (16, 17). Chen went on to demonstrate that many human cancers upregulate PD-L1 and that blocking of the PD-L1/PD-1 interaction by antibodies leads to tumor regression in mice (18). These discoveries paved the way for the clinical success of PD-1 blockade in treating advanced solid tumors (19-21). The Review by Chen and Han focuses on the history and current developments of anti-PD-1 therapy (22). They also discuss basic characteris- tics of anti-PD-1 therapy and how this therapy is distinct from the anti-CTLA-4 approach.

\section{CAR T cell adoptive immunotherapy}

CAR technology was first reported in 1993 by Zelig Eshhar and colleagues, who transduced $\mathrm{T}$ cells with chimeric genes encoding single-chain antibodies linked to a transmembrane region and an intracellular domain encoding the signaling adaptor for the $\mathrm{T}$ cell receptor (23). It was demonstrated that CAR T cell therapy could redirect $\mathrm{T}$ cell killing to cells expressing the antibody's cognate antigen. Later, it was shown that CD19 CAR-transduced human peripheral blood T lymphocytes could eradicate lymphoma and leukemia in immune-deficient mice (24). In 2010, a case report showed an encouraging result using CD19 CAR T cells for treating a patient with lymphoma (25). Since then, CAR T cell therapies have shown impressive clinical outcomes in treating patients with relapsed or refractory B cell malignancies, including acute and chronic lymphocytic leukemia (26-28). CAR T cells targeting solid tumors have also been tested but have only yielded modest results so far. Here, using the CD19 paradigm, Sadelain provides a comprehensive review on the development of CAR technology, its clinical efficacy in treating B cell malignancies, and lessons learned from CD19 CAR therapy as well as potential CAR therapy-associated toxicities (29).

\section{Therapeutic cancer vaccines and tumor neoantigens}

Despite impressive clinical outcomes achieved with immune checkpoint blockade and CAR therapies, the overall results of therapeutic vaccination against established tumors remain suboptimal, as clinical benefit for patients with cancer was largely noted as prolonged survival. The Review by Melief and colleagues explains that the reasons for lack of cancer eradication are suboptimal vaccine design and the presence of an immunosuppressive tumor microenvironment (30). They further discuss how better results may be obtained by improvements in antigen choice and vaccine design as well as appropriate treatments that reverse immunosuppressive mechanisms, such as PD-1 blockade.

Among antigen choices, neoantigens that arise as a consequence of tumor-specific mutations have been postulated to be of particular relevance to the control of cancer upon vaccination because $\mathrm{T}$ cells for these antigens are not deleted by central tolerance mechanisms (31). However, the identification of tumor neoantigens has historically been time consuming and labor intensive. The review by Gubin and colleagues discusses recent advances in next-generation sequencing and epitope prediction that make the rapid identification of tumor neoantigens possible (32). They also discuss the use of tumor neoantigens in personalizing cancer immunotherapies.

\section{Conclusions}

In summary, this Review Series surveys recent advances in cancer immunology and immunotherapy and highlights the breakthrough immune checkpoint and CAR T cell therapies, which mark the beginning of a new era in cancer immunotherapy. Despite this success, immunotherapy only works in a subset of cancers, and only a fraction of patients with cancer respond to immunotherapy. Additionally, better clinical response by therapeutic cancer 
vaccines is desired. The development of immune checkpoint blockade and CAR T cell therapies underscores the importance of understanding basic tumor immunology, particularly the roles of the immunosuppressive tumor microenvironment and tumor neoantigens in shaping the development of cancer and influencing therapeutic efficacy. Continued efforts to improve our understanding of tumor immunology will provide important insights into developing more effective therapies.

\section{Acknowledgments}

The author would like to acknowledge the support of NIH grants R01 CA136934 and R21 CA186973.

Address correspondence to: Yiping Yang, Department of Medicine, Duke University Medical Center, Box 103005, Durham, North Carolina 27710, USA. Phone: 919.668.0932; E-mail: yang0029@mc.duke.edu.
1. Drake CG, Jaffee E, Pardoll DM. Mechanisms of immune evasion by tumors. Adv Immunol. 2006;90:51-81.

2. Couzin-Frankel J. Breakthrough of the year 2013. Cancer immunotherapy. Science. 2013;342(6165):1432-1433.

3. Schreiber RD, Old LJ, Smyth MJ. Cancer immunoediting: integrating immunity's roles in cancer suppression and promotion. Science. 2011;331(6024):1565-1570.

4. Teng MWL, Galon J, Fridman W-H, Smyth MJ. From mice to humans: developments in cancer immunoediting. J Clin Invest. 2015; 125(9):3338-3346.

5. Shalapour S, Karin M. Immunity, inflammation, and cancer: an eternal fight between good and evil. J Clin Invest. 2015;125(9):3347-3355.

6. Marvel D, Gabrilovich DI. Myeloid-derived suppressor cells in the tumor microenvironment: expect the unexpected. JClin Invest. 2015;125(9):3356-3364.

7. Ugel S, De Sanctis F, Mandruzzato S, Bronte V. Tumor-induced myeloid deviation: when myeloid-derived suppressor cells meet tumor-associated macrophages. J Clin Invest. 2015;125(9):3365-3376.

8. Brunet JF, et al. A new member of the immunoglobulin superfamily - CTLA-4. Nature. 1987;328(6127):267-270.

9. Walunas TL, et al. CTLA-4 can function as a negative regulator of $\mathrm{T}$ cell activation. Immunity. 1994;1(5):405-413.

10. Krummel MF, Allison JP. CD28 and CTLA-4 have opposing effects on the response of T cells to stimulation. J Exp Med.1995;182(2):459-465.

11. Tivol EA, Borriello F, Schweitzer AN, Lynch WP, Bluestone JA, Sharpe AH. Loss of CTLA-4 leads to massive lymphoproliferation and fatal multiorgan tissue destruction, revealing a critical negative regulatory role of CTLA-4. Immunity. 1995;3(5):541-547.

12. Waterhouse $P$, et al. Lymphoproliferative disorders with early lethality in mice deficient in Ctla-4. Science. 1995;270(5238):985-988.

13. Leach DR, Krummel MF, Allison JP. Enhancement of antitumor immunity by CTLA- 4 blockade. Science. 1996;271(5256):1734-1736.

14. Buchbinder E, Hodi FS. Cytotoxic T lymphocyte antigen-4 and immune checkpoint blockade. J Clin Invest. 2015;125(9):3377-3383.

15. Ishida Y, Agata Y, Shibahara K, Honjo T. Induced expression of PD-1, a novel member of the immunoglobulin gene superfamily, upon programmed cell death. EMBO J. 1992;11(11):3887-3895.

16. Dong H, Zhu G, Tamada K, Chen L. B7-H1, a third member of the B7 family, co-stimulates T-cell proliferation and interleukin-10 secretion. Nat Med.1999;5(12):1365-1369.

17. Freeman GJ, et al. Engagement of the PD-1 immunoinhibitory receptor by a novel B7 family member leads to negative regulation of lymphocyte activation. J Exp Med. 2000;192(7):1027-1034.

18. Dong H, et al. Tumor-associated B7-H1 promotes T-cell apoptosis: a potential mechanism of immune evasion. Nat Med. 2002;8(8):793-800.

19. Wolchok JD, et al. Nivolumab plus ipilimumab in advanced melanoma. $N$ Engl J Med. 2013;369(2):122-133.

20. Brahmer JR, et al. Safety and activity of antiPD-L1 antibody in patients with advanced cancer. N Engl J Med. 2012;366(26):2455-2465.

21. Topalian SL, et al. Safety, activity, and immune correlates of anti-PD-1 antibody in cancer. $N$ Engl JMed. 2012;366(26):2443-2454.

22. Chen L, Han X. Anti-PD-1/PD-L1 therapy of human cancer: past, present, and future. J Clin Invest. 2015;125(9):3384-3391.
23. Eshhar Z, Waks T, Gross G, Schindler DG. Specific activation and targeting of cytotoxic lymphocytes through chimeric single chains consisting of antibody-binding domains and the gamma or zeta subunits of the immunoglobulin and T-cell receptors. Proc Natl Acad Sci U S A. 1993;90(2):720-724.

24. Brentjens RJ, et al. Eradication of systemic B-cell tumors by genetically targeted human $\mathrm{T}$ lymphocytes co-stimulated by CD80 and interleukin- 15 . Nat Med. 2003;9(3):279-286.

25. Kochenderfer JN, et al. Eradication of B-lineage cells and regression of lymphoma in a patient treated with autologous T cells genetically engineered to recognize CD19. Blood. 2010;116(20):4099-4102.

26. Kalos M, et al. T cells with chimeric antigen receptors have potent antitumor effects and can establish memory in patients with advanced leukemia. Sci Transl Med. 2011;3(95):95ra73.

27. Grupp SA, et al. Chimeric antigen receptor-modified T cells for acute lymphoid leukemia. $N$ Engl J Med. 2013;368(16):1509-1518.

28. Brentjens RJ, et al. CD19-targeted T cells rapidly induce molecular remissions in adults with chemotherapy-refractory acute lymphoblastic leukemia. Sci Transl Med. 2013;5(177):177ra38.

29. Sadelain M. CAR therapy: the CD19 paradigm. JClin Invest. 2015;125(9):3392-3400.

30. Melief CJM, van Hall T, Arens R, Ossendorp F, van der Burg SH. Therapeutic cancer vaccines. J Clin Invest. 2015;125(9):3401-3412.

31. Schumacher TN, Schreiber RD. Neoantigens in cancer immunotherapy. Science. 2015;348(6230):69-74.

32. Gubin MM, Artyomov MN, Mardis ER, Schreiber RD. Tumor neoantigens: building a framework for personalized cancer immunotherapy. J Clin Invest. 2015;125(9):3413-3421. 